\title{
Extracellular matrix remodeling in equine sarcoid: an immunohistochemical and molecular study
}

\author{
Manuela Martano ${ }^{1 *}$, Annunziata Corteggio ${ }^{2}$, Brunella Restucci ${ }^{1}$, Maria Ester De Biase ${ }^{1}$, Giuseppe Borzacchiello \\ and Paola Maiolino ${ }^{1}$
}

\begin{abstract}
Background: Equine sarcoids are locally invasive, fibroblastic benign skin tumors. Bovine papillomavirus type-1 (BPV-1) and/or Bovine papillomavirus type-2 (BPV-2) are believed to be the causative agent of sarcoids, although the mechanisms by which the virus induce the tumor are still poorly understood. We hypothesized that in genetically predisposed equines latent BPV infection may be reactivated by immunosoppression and/or mechanical injury leading to a form of pathologic wound which may transform into a sarcoid. In this study, we investigated in 25 equine sarcoids and in five normal skin samples the histological features and evaluated the immunohistochemical and molecular expression of type I and type III Collagen, vimentin (VIM), alfa Smooth Muscle Actin (a-SMA), Matrix Metalloproteinase (MMPs) -2, 9, 14 and tissue inhibitor of metalloproteinase 2 (TIMP-2).

Results: In $64 \%$ of investigated sarcoids, type I collagen staining was stronger than that of type III collagen. In $80 \%$ of sarcoids, SFs were strongly positive for vimentin and negative for a-SMA; the remaining sarcoid samples (20\%) showed 70-80 \% of SFs labeled for vim and approximately 20-30\% labeled for a-SMA. Moreover, all sarcoid specimen showed a variable staining pattern (weak to moderate) for MMP-9 and MMP-14, and a moderate to strong staining for MMP-2 and TIMP-2. Biochemical analysis confirmed immunohistochemical results and showed in sarcoids, for the first time, the cleaved form of MMP9, the $35 \mathrm{KDa}$ active species for MMP-9.

Conclusions: This study revealed that in equine sarcoids exhibit an altered turnover of the Extracellular Matrix (ECM) deposition and degradation, as result of an altered expression of MMPs and TIMPs. Therefore, these observations seem to confirm that the basic mechanism for growth of equine sarcoids could be a neoplastic transformation during wound healing.
\end{abstract}

Keywords: BPV, ECM, Equine sarcoid, MMPS

\section{Background}

Equine sarcoids are locally invasive, fibroblastic benign skin tumors and represent the most common skin tumor in equidae worldwide $[1,2]$. They can occur as single lesion, or, more commonly, as multiple lesions, frequently at sites of previous injury and scarring; although they can develop anywhere on the integument sites of predilection are in particular the paragenital region, the thorax-abdomen and the head [3, 4]. Equine sarcoids

\footnotetext{
*Correspondence: manuela.martano@unina.it

'Department of Veterinary Medicine and Animal Productions, Naples

University "Federico II", Via F. Delpino 1, 80137 Naples, Italy

Full list of author information is available at the end of the article
}

rarely regress, are notoriously difficult to treat and are associated with a high recurrence rate following surgical intervention [5-7]; these features are likely due to the invasiveness of sarcoid fibroblasts (SFs) $[8,9]$. BPV-1 and less commonly BPV-2 are widely recognized as the causative agents of the disease. Although the viral etiology, the biology, the morphology and the epidemiology of equine sarcoids are known $[4,10,11]$, the pathogenic events leading to the development of tumour and the mechanisms used by BPV to induce the tumour are less understood. Sarcoid formation is known as one of the main long-term complications in the wound healing of horses [12, 13]. We hypothesized that in healthy 
genetically predisposed horses, BPV-1/BPV2 may be responsible for abnormal fibroblast proliferation on one hand, and on the other for alterations in dynamics of the extracellular matrix (ECM) and its main components (e.g. collagen). These changes could induce an alteration of the wound healing process and may therefore be an important factor in the pathogenesis of equine sarcoids. The hypothesis that cancer may be "a wound that won't heal" has been supported by numerous studies [14-16] suggesting that wound healing and tumorigenesis share consistently similarities in terms of histological features and signaling molecules; those are among others Matrix Metalloproteinases (MMPs), a family of at least 25 zinc-dependent endopeptidases, and their inhibitors (TIMPs) all essentially capable of degrading Extracellular Matrix (ECM), including collagen. Besides participating in normal connective tissue homeostasis and remodeling, MMPs activity is involved in remodeling of ECM and the migration of numerous cell types during various pathological conditions, such as wound healing, keloid formation, chronic inflammatory diseases, and as well as in tumour invasion [17-19]. In this study, we speculate that changes of the expression levels and of the enzymatic activity of MMP-2, MMP-9, MMP-14 (MMP1MMT) and TIMP-2 may play an important role in the pathogenesis of sarcoids, being responsible for ECM turnover. Of the growing family of MMPs, MMP-2 (gelatinase A, 72-kDa type IV collagenase,) and MMP-9 (gelatinase B, 92-kDa type IV collagenase) are unique for their fibronectin-like collagen binding domains and are responsible of degradation of type IV collagen in the basement membranes and in fibrillar collagens, which are essential features of tissue repair and remodeling processes. Their activity is controlled by a group of protein inhibitors, the TIMPs. MMP-14 is a trans-membrane protease, capable of degrading different ECM components such as collagen types I, II, and III, as well as fibronectin and laminin [20]. The main interest in this enzyme is due to its ability to activate different proteases, particularly MMP-2 and MMP9 [21]. Recently, Yuan et al. (2010) [8] have shown that BPV-1 induce overexpression of MMPs contributing to invasiveness of SFs in vitro. Our in vivo study aimed at gaining new insights into the pathogenetic mechanisms of equine sarcoids, by employing immunohistochemistry and western blot analysis to investigate their histological features, as well as the expression of type I and type III collagen, MMP-2, MMMP-9, MMP-14 and their inhibitors, such as TIMP-2. Moreover, the enzymatic activities of MMP-2 and MMP-9 were quantified by gelatinzymography of the same homogenized tumour tissues.

\section{Results}

\section{Histological features}

Examined sarcoids showed the typical histological changes in their epidermal (when present) and dermal component such as hyperkeratosis and epidermal hyperplasia often accompanied by rete pegs extending deep into the proliferating dermal connective tissue. Dermal proliferation consisted of tightly whirling plump spindle cells, proliferating in an ECM which appeared more developed than normal. The superficial dermal fibroblasts were usually oriented perpendicular to the basilar epidermal layer in a 'picket fence' pattern (Fig.1a). Ulceration as well as inflammation (infiltration of polymorphonuclear cells) were commonly seen. Van Gieson's stain of sarcoids confirmed an increase in the amount of deep red color collagen fibers in the dermis, identified as mature collagen (Type I), compared with pink color collagen fibers, identified as immature collagen (Type III) (Fig.1b), as compared to normal skin.

\section{Immunohistochemistry}

The expression patterns of Vimentin (VIM), alphaSmooth Muscle Actin ( $\alpha$-SMA), type I Collagen, type III Collagen, MMP-2, MMP-9, MMP-14, and TIMP-2 in 25 equine sarcoids and five normal skin samples are summarized in Table 1.

\section{Normal skin}

All normal skin samples showed positive immunostaing for type I and III Collagen, which was light brown stained with a widely distributed staining pattern within the dermal layer. Moreover, a weak and finely granular cytoplasmic MMP-2 and MMP-9 reactivity was observed in the epidermis. TIMP-2 and MMP-14 immunoexpression was present in the epidermis but also in vascular endothelial cells, inflammatory cells and fibroblastic cells.

\section{Sarcoid samples}

Type I and Type III collagen appeared as fine discontinuous individual fibers in a loose network, and showed a moderate immunosignal in $36 \%$ of sarcoid samples (Fig. 2: a-b). Type I Collagen staining was stronger than type III collagen in the remaining samples (64\%). In $80 \%$ of sarcoids, SFs were strongly positive for vimentin and negative for $\alpha$-SMA; in the remaining samples (20 \%), 70-80 \% of SFs were labeled for vimentin (Fig. 3a) and approximately 20-30 \% were strongly labeled for $\alpha$-SMA (Fig. 3b). Furthermore, sarcoid specimens showed a variable (64\% moderate; $36 \%$ strong) and finely granular staining pattern for MMP-2 in 30$50 \%$ of SFs, as well as in the cytoplasm of epidermal cells (Fig. 4a). Sarcoids featured a weak (52\%) to moderate (48 \%) staining for MMP-9, which appeared highly and finely granular in the cytoplasm of epidermal cells and rarely (often lacking) in the rete peg epithelium. A moderate to strong cytoplasmic staining for MMP-9 was observed in 30-70\% of SFs, inflammatory cells and vascular endothelial cells (Fig. 5a). TIMP-2 showed 


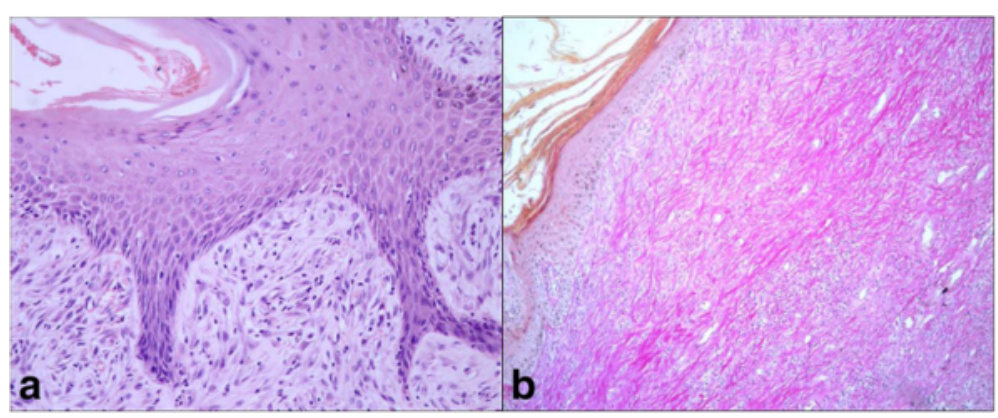

Fig. 1 a Equine sarcoid. Epidermal hyperplasia, rete peg and picket fence formation. Hematoxylin-eosin. 20X. b Equine sarcoid. Red color collagen fibers (collagen I) in equine sarcoid. Van Gieson Stain. 10X

Table 1 Immunoreactivity scoring of VIM, a-sma, Type I collagen, Type III collagen, MMP-2, MMP-9, MMP14, TIMP-2 in 25 equine sarcoids and 5 normal skin

\begin{tabular}{|c|c|c|c|c|c|c|c|c|c|}
\hline Samples $^{a}$ & Location & $\mathrm{VIIM}^{\mathrm{b}}$ & $A-S M A^{b}$ & Type I collagen ${ }^{b}$ & Type III collagen b & $M M P-2^{b}$ & MMP-9 $9^{b}$ & MMP14 & $\mathrm{TIMP}^{\mathrm{b}} \mathrm{2}^{\mathrm{b}}$ \\
\hline $\mathrm{T} 1$ & Abdomen & ++ & - & + & + & + & + & $+/-$ & ++ \\
\hline $\mathrm{T} 2$ & Limbs & ++ & - & + & + & ++ & + & $+/-$ & ++ \\
\hline T3 & Neck & ++ & - & + & + & + & + & $+/-$ & ++ \\
\hline T4 & Abdomen & ++ & - & + & + & + & $+/-$ & $+/-$ & ++ \\
\hline T5 & Paragenit region & ++ & - & + & + & ++ & + & + & ++ \\
\hline T6 & Pectoral region & ++ & - & + & + & + & $+/-$ & + & ++ \\
\hline T7 & Paragenit region & ++ & - & + & + & + & $+/-$ & + & ++ \\
\hline T8 & Neck & ++ & - & + & + & ++ & + & + & ++ \\
\hline T9 & Neck & ++ & - & + & + & + & $+/-$ & $+/-$ & ++ \\
\hline $\mathrm{T} 10$ & Pectoral region & ++ & - & ++ & + & + & + & $+/-$ & ++ \\
\hline T11 & Paragenit region & ++ & - & ++ & + & ++ & + & $+/-$ & ++ \\
\hline $\mathrm{T} 12$ & Limbs & ++ & - & ++ & + & ++ & + & $+/-$ & ++ \\
\hline $\mathrm{T} 13$ & Neck & ++ & - & ++ & + & + & $+/-$ & + & ++ \\
\hline T14 & Abdomen & ++ & - & ++ & + & + & $+/-$ & + & ++ \\
\hline T15 & Limbs & ++ & - & ++ & + & ++ & + & $+/-$ & ++ \\
\hline T16 & Pectoral region & ++ & - & ++ & + & + & $+/-$ & $+/-$ & ++ \\
\hline T17 & Pectoral region & ++ & - & ++ & + & + & $+/-$ & $+/-$ & ++ \\
\hline T18 & Limbs & ++ & - & ++ & + & ++ & + & + & ++ \\
\hline T19 & Abdomen & ++ & - & ++ & + & + & $+/-$ & + & + \\
\hline T20 & Pectoral region & ++ & - & ++ & + & + & $+/-$ & $+/-$ & + \\
\hline T21 & Limbs & ++ & ++ & ++ & + & ++ & + & $+/-$ & + \\
\hline T22 & Neck & ++ & ++ & ++ & + & ++ & + & $+/-$ & + \\
\hline $\mathrm{T} 23$ & Abdomen & ++ & ++ & ++ & + & + & $+/-$ & + & + \\
\hline T24 & Paragenit region & ++ & ++ & ++ & + & + & $+/-$ & + & + \\
\hline T25 & Pectoral region & ++ & ++ & ++ & + & + & $+/-$ & + & + \\
\hline N1 & Limbs & n.a. & n.a. & $+/-$ & $+/-$ & + & $+/-$ & - & - \\
\hline N2 & Abdomen & n.a. & n.a. & $+/-$ & $+/-$ & + & $+/-$ & $+/-$ & $+/-$ \\
\hline N3 & Pectoral region & n.a. & n.a. & $+/-$ & $+/-$ & + & $+/-$ & - & $+/-$ \\
\hline N4 & Neck & n.a. & n.a. & $+/-$ & $+/-$ & + & $+/-$ & $+/-$ & - \\
\hline N5 & Limbs & n.a. & n.a. & $+/-$ & $+/-$ & + & $+/-$ & $+/-$ & $+/-$ \\
\hline
\end{tabular}

${ }^{\mathrm{a}} T$ tumour sample, $N$ normal skin sample, ${ }^{\mathrm{b}}$ - negative staining; +/- weak immunolabelling; + moderate immunolabelling; ++ extensive and strong immunolabelling; n.a. not assessed 


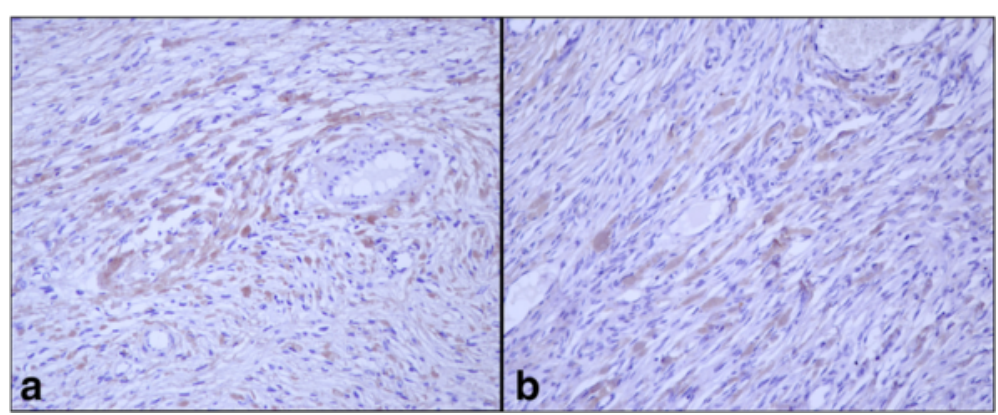

Fig. 2 a Type I Collagen and b Type III collagen immunostaining in equine sarcoids. Streptavidin-biotin-peroxidase stain 20X

extensive and strong cytoplasmic positivity in epidermal cells and in 50-70 \% of SFs in almost every sample (72 \%) (Fig. 6a). In all tumors, MMP-14 expression was observed in epidermis and in 50-70 \% of SFs, with variable (56 \% weak; $44 \%$ moderate) cytoplasmic immunolabelling (Fig. 7a).

\section{Biochemical analysis}

To further confirm our findings, six sarcoid samples, which were available for biochemical analysis and two skin samples from healthy horses were subjected to western blot analysis. Hela cell line was also analyzed as positive control for the antibodies used (data not shown). The anti-Collagen I and anti-Collagen III antibodies yielded a band of the expected molecular weight in the neoplastic tissues and normal skin. An increase in the amount of both collagen type protein levels in all tumour samples compared with normal skin was observed, albeit in different amounts among the samples (Fig. 8). The analysis of MMP-2 showed that $50 \%$ of sarcoid samples (T3, T4 and T5) had a high level of protein, above all in its active (cleaved) form $(62 \mathrm{kDa})$. In addition, an increase in the expression level of MMP9 in all tumour samples versus healthy skin samples was observed. The sarcoids showed the overexpression of the cleaved form of MMP9, the $35 \mathrm{KDa}$ active species which was an autocatalytic product of the $82 \mathrm{KDa}$ pre-form.
We analyzed the expression of MMP-14 and TIMP-2, in order to evaluate their involvement in MMP2 activity. There was no significant up-regulation of MMP14 protein in the sarcoids compared to normal skin samples, even if in one sarcoid sample (T1) it was present at a very high level. TIMP2 was overexpressed in sarcoids when compared to normal skin. Actin was shown as control for ensuring the equal loading of protein extracts (Fig. 9). Finally, we examined gelatinase MMP's activity in sarcoid tumors using zymography, which has been extensively used to detect both latent and active form of MMPs. Gelatine zymography was employed to specifically detect MMP-2 and MMP-9 protease activity. As shown in Fig. 10, all the examined sarcoids expressed the pro- and active form of MMP2. However, sarcoids showed higher expression levels than normal skin samples. In addition, the MMP9 was present in the activated form in all the analyzed sarcoids. Interestingly, four out of six the sarcoid samples ( $80 \%)$ overexpressed a cleaved form of $35 \mathrm{kDa}$ MMP9, thus demonstrating the strong activity of MMP9 during sarcoids tumorigenesis.

\section{Discussion}

Sarcoids are the most common equine skin tumours, characterized by neoplastic fibroblasts intermingled in a collagenous stroma, frequently associated to epidermal hyperplasia [2, 22]. BPV-1/BPV-2 are believed to be the

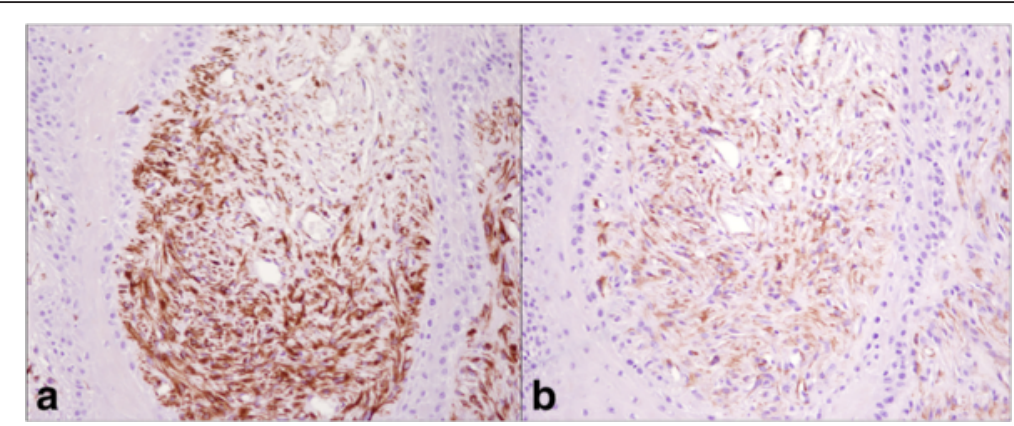

Fig. 3 VIM and Alfa-sma immunostaining in equine sarcoid. a 70-80 \% of SFs show strong vimentin immunostaining; b 20-30 \% of SFs show strong Alfa-sma immunostaining. Streptavidin-biotin-peroxidase stain 20X 


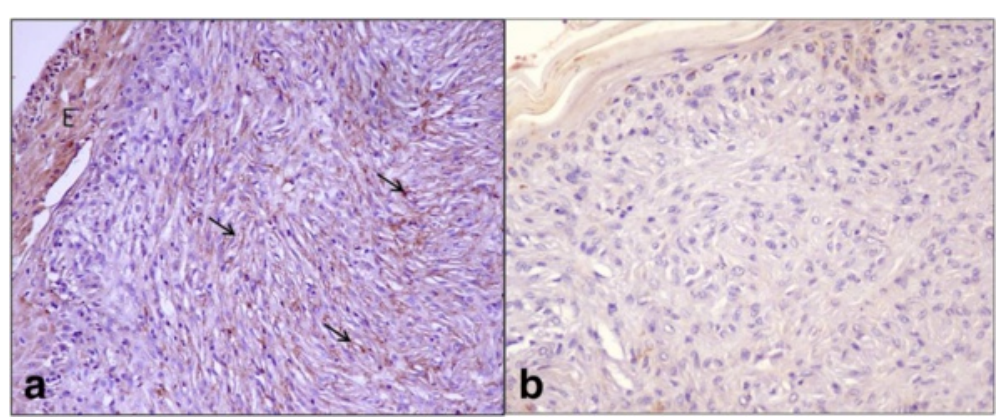

Fig. 4 a MMP-2 immunostaining in equine sarcoid. SFs (arrows) show a strong immunostaining, the epidermis (E) is also strongly MMP-2 positive. b Secondary-only negative control. Streptavidin-biotin-peroxidase stain. 20X

causative agent of equine sarcoid. This is based on the fact that: 1) BPV-1/-2 DNA is detected in the majority of sarcoid tumors [7, 23-27]; 2) BPV genes are expressed in sarcoids $[10,28-30]$; 3) experimental inoculation of equine skin with BPV induces sarcoid-like lesions in horses [31]; 4) BPV- 1 DNA can transform primary equine fibroblasts in vitro [8,32]. Interestingly, BPV DNA presence has also been reported in normal skin and the virus has been found to be transcriptionally active in some cases of equine inflammatory skin lesions [33, 34]. It is noteworthy that very recently BPV has been found also in exuberant granulation tissue [35]. It is widely accepted that equine sarcoids may develop subsequently to injury and scarring in genetically predisposed equines [24]. For this reason we hypotized that latent BPV infection may be reactivated by chronic physical trauma, leading to development of a form of pathologic wound healing (e.g. keloid); thus, the scar producing process may be altered during the maturation phase of wound healing allowing transformation of scar tissue (keloids) into sarcoids.

In fact, our immunohistochemical results showed that, in most sarcoids (20/25), fibroblasts represented the principal cellular population, the remainder was composed of fibroblasts and myofibroblasts.
Furthermore, in our sample tissues collagen content was elevated and disorganized when compared to normal skin and, in contrast to what Williams et al. reported [36], the ratio of type I (mature collagen) to type III collagen (immature collagen) seemed to be slightly higher, which was as also demonstrated by Van Gieson stain. Also, and in line with the immunohistochemical results, western blotting analysis confirmed that collagen I and III were present in higher amounts in tumour samples when compared to normal skin samples. It is known tissue of normal wound repair contains primarily type III collagen with abundant myofibroblasts. In contrast, abnormal wound repair tissue, (for instance keloid), consists of type I and III bundles with few myofibroblasts [37]. Combining these data strongly suggests that these sarcoid tumours actually originate in abnormal wound repair tissue [38].

We hypothesized that the basic mechanism for the development of equine sarcoids could be an imbalance of ECM deposition and degradation as seen also during pathologic wound healing.

This process is mediated by ECM degrading enzymes, such as MMPs, and we believe that these changes are likely the result of altered expression levels between these enzymes and their inhibitors (TIMPs). The MMPs

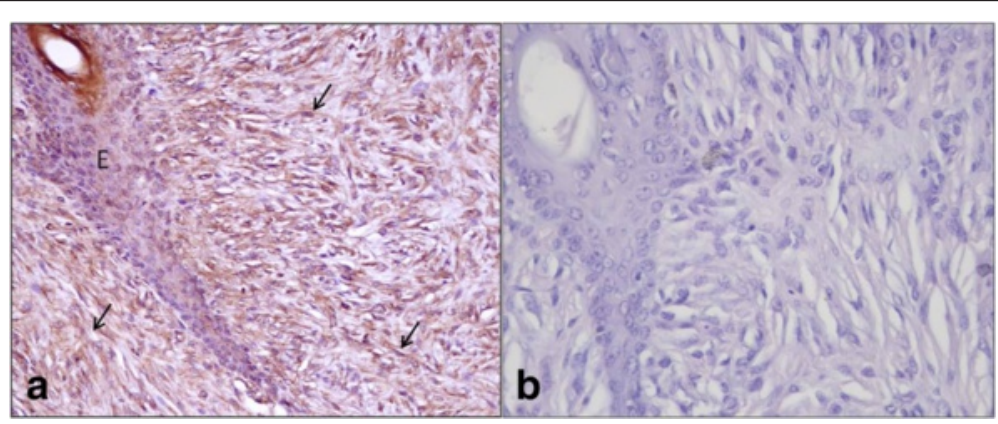

Fig. 5 a MMP-9 immunostaining in equine sarcoid. The epidermis (E) shows strong positivity except for rete peg epithelium, SFs are strongly MMP-9 positive (arrows). b Secondary-only negative control. Streptavidin-biotin-peroxidase stain. 20X 


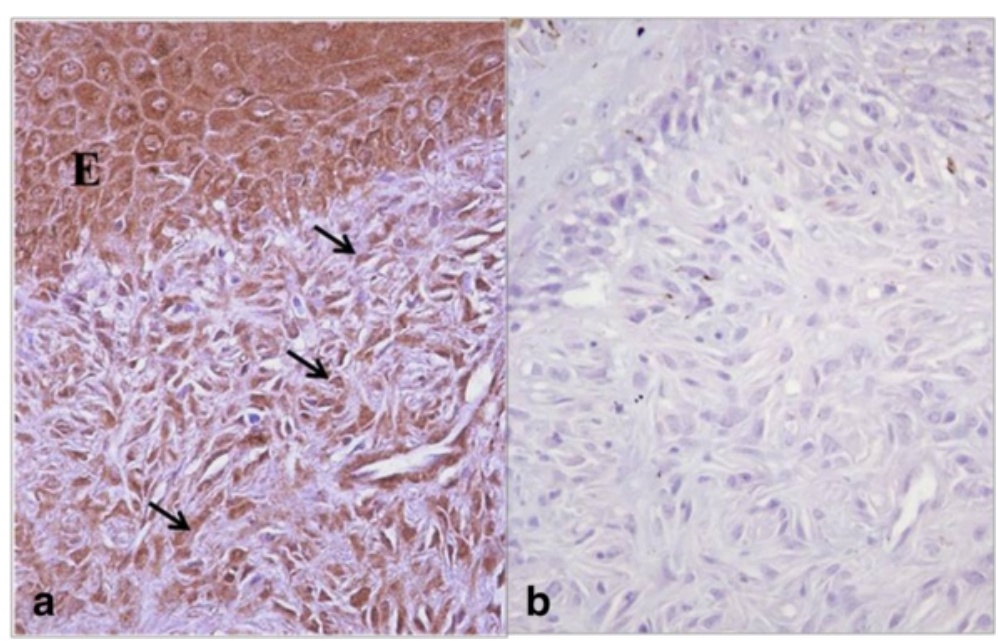

Fig. 6 a TIMP-2 immunostaining in equine sarcoid. TIMP-2 is strongly expressed by epidermis (E) and by 50-70\% of SFs (arrows). b Secondary-only negative control. Streptavidin-biotin-peroxidase stain. 40X

are usually not detectable or at very low levels in healthy resting tissue and are instead induced in wound repair and in keloid formation in response to cytokines, growth factors and/or cell contact with ECM [17-19]. It has been shown that the over-expression and activation of MMPs is induced by BPV oncoproteins in equine sarcoid fibroblasts and, recently, MMPs expression has been confirmed also in vivo $[8,9,32]$. These previous observations are in line with the results of our study in which we also found that both MMP-9 and MMP-2 were consistently expressed by epidermal and dermal cells and with different intensity.

MMP-9 is secreted as pro-MMP-9 $(92 \mathrm{kDa})$ and is activated into the functional form $(82 \mathrm{kDa})$.

In equine sarcoids our data show for the first time, the overexpression of a $35 \mathrm{KDa}$ super-active form of MMP-9, an auto-catalytic product of the $82 \mathrm{KDa}$ proform, which possesses highly efficient proteolytic activity for different ECM proteins (gelatins, fibronectin and collagen type IV) [39]. In our sarcoid samples, MMP-9 was expressed by
SFs and keratinocytes, strongly suggesting its role in the formation of long rete pegs [40, 41]; this in turn promotes keratinocyte detachment from the basement zone through controlled digestion of type IV collagen.

Thus, sarcoid development could be the result of fibroblast stimulated proliferation of overlying epithelial cells (with rete peg formation), which in turn stimulate the fibroblasts in the underlying dermis to proliferate and to produce more collagen.

In our study, MMP-2 showed a variable and finely granular staining pattern in 30-50 \% of SFs, confirming that fibrillar collagens, rather that collagen IV, are its specific substrate.

Normally, MMP-2 is secreted as pro-MMP-2 $(72 \mathrm{kDa})$ and is activated into the functional form $(66 \mathrm{kDa})$. Biochemical analysis showed that MMP-2 was overexpressed and hyper-activated in $50 \%$ of sarcoid samples, when compared to normal skin. Moreover, in sarcoids MMP-2 expression was associated with a weak to moderate expression of MMP-14, which is considered its

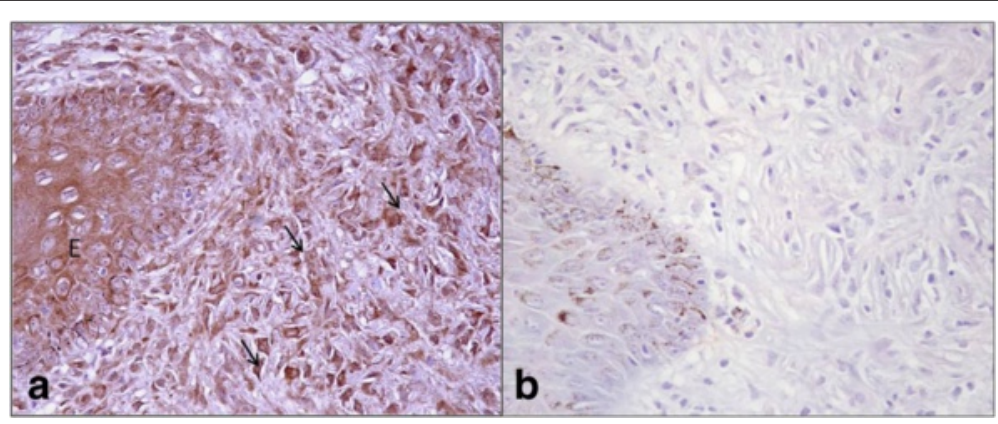

Fig. 7 a MMP-14 immunostaining in equine sarcoid. MMP-14 is strongly stained by epidermis (E) and by $50-70 \%$ of SFs (arrows). b Secondary-only negative control. Streptavidin-biotin-peroxidase stain. 40X 


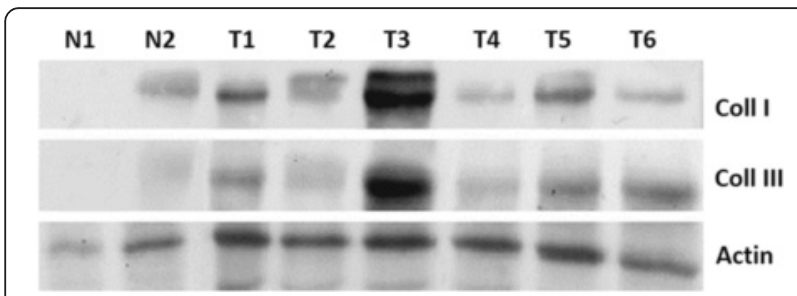

Fig. 8 Collagen type I and type III protein expression in equine sarcoids (T) and normal skin (N). Collagen type III are expressed in higher amount in sarcoids ( $T$ ) when compared to normal skin samples (N). Actin protein levels confirm the amount of protein loading in each lane

main activator; this, with a strong expression of TIMP-2, which is considered its main inhibitor [42]. Although MMP-14 expression seems increased, the higher level of TIMP-2, could lead to a decrease in MMP2 collagenolytic activity, which in turn causes insufficient degradation of collagen produced in excess by SFs. In fact, in our sarcoid samples a high content of collagen(s) was observed, suggesting that ECM deposition continues with insufficient degradation.

Therefore, we postulate that excessive and progressive deposition of connective tissue (collagen) in sarcoids, as well as in keloids [43], might not only be the result of elevated synthesis by SFs, but also caused by a deficiency in matrix degradation due to an alterated expression of MMPs and TIMPs. This imbalance between production and degradation of collagen could play an important role in the pathogenesis of the equine sarcoid. Therefore, it may be suggested that, in genetically predisposed

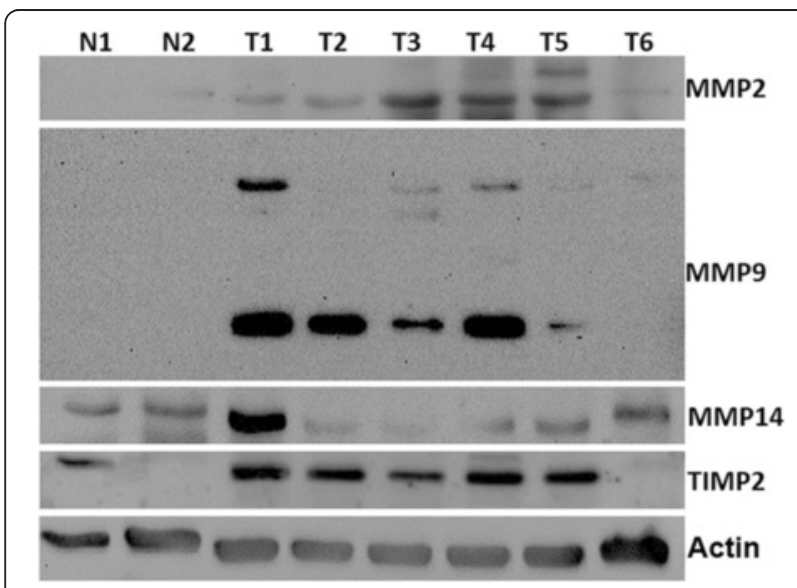

Fig. 9 MMP-2, MMP-9, MMP-14 and TIMP-2 protein expression in equine sarcoids (T) and normal skin (N). MMP-2 and MMP-9 are expressed in higher amount in sarcoids. MMP9 is present in two different forms, a 82 kDa band and 35 kDa band (super active form). MMP-9 is expressed at similar levels in all the analyzed samples, albeit in T1 samples is present at very higher level. TIMP-2 is expressed in higher amount in sarcoids (T) when compared to normal skin samples (N). Actin protein levels confirm the equal amount of protein loading in each lane

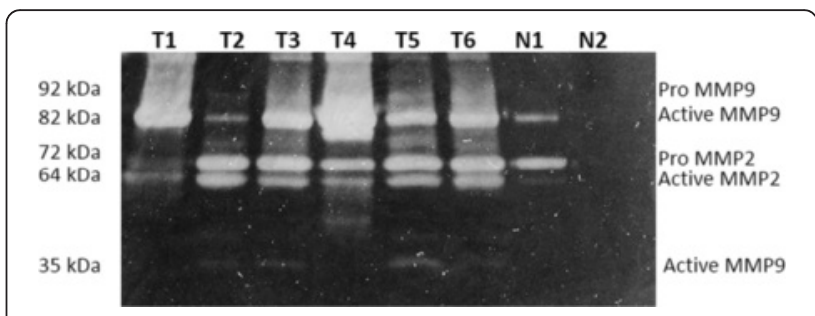

Fig. 10 MMP-2 and MMP-9 protein expression and activation. Gelatin zymography was performed from equine sarcoids (T) and normal skin (N) tissues homogenates supernatants. In all sarcoids the active form of MMP-9 (82 kDa) is the predominant form, and a super active form (35 kDa) is present in the same samples. MMP-2 is present in both proform (72 kDa) and active form (62 kDa) in all sarcoids

equines with latent BVP infection, an altered wound healing process creates a microenvironment that activates the latent infection, leading to neoplastic transformation and sarcoid formation.

\section{Conclusions}

Currently, there is no efficient curative therapy for equine sarcoids. The commonly employed treatments include cryotherapy, surgical excision and local immune modulation [44]. The present findings include the identification of the main cellular effectors of sarcoid growth, that is, the key cytokines regulating the scar formation process, and the regulators of ECM turnover; these findings therefore open the avenue for a number of potential therapeutic approaches that are likely to be developed in the near future.

\section{Methods}

Tumour samples

Ethics approval was obtained from the Ethical Animal Care and Use Committee of the University of Naples Federico II (Prot. N $\left.{ }^{\circ} 125861 ; 23 / 12 / 2015\right)$.

Twenty-five samples of equine sarcoid (each from a different horse), were clinically identified based on their gross morphology according to Pascoe and Knottenbelt (1999) [45]. Tumors were localized on the abdomen (5), neck (5), (para)-genital (4), pectoral region (6), and limbs (5) (Table 1). The length of time the sarcoids were present was variable (from 1 month to over 6 years), and often not precisely known.

No history of previous skin lacerations (wounds) could be determined due to lack of informations from both owners and practioneers. Sarcoid tissues used in this study were known to be positive for BPV 1- BPV 2 DNA ([28]; personal observations). Tumors, together with five normal skin samples from healthy horses, were either surgically excised or a representative biopsy was taken under local anesthesia. In all cases, owners signed a written consent form following a detailed verbal explanation of the study protocol. Samples were $10 \%$ formalin fixed, 
paraffin-embedded for routine histological processing and stained with haematoxylin and eosin for light microscopy study and Verhoeff-Van Gieson (VVG) method to asses collagen content.; six out of 25 sarcoid samples were perfused thoroughly with cold $0.9 \% \mathrm{NaCl}$ and frozen at $-80{ }^{\circ} \mathrm{C}$ for western blotting analysis.

\section{Immunohistochemistry}

Paraffin sections of 25 sarcoids and five normal skin from healthy horses were dewaxed in xylene, dehydrated in graded alcohols and washed in $0.01 \mathrm{M}$ phosphate-buffered saline (PBS), $\mathrm{pH}$ 7.2-7.4. Endogenous peroxidase was blocked with hydrogen peroxide $0.3 \%$ in absolute methanol for $30 \mathrm{~min}$. The immunohistochemical procedure (streptavidin biotin- peroxidase method, (LSAB Kit; Dako, Glostrup, Denmark) was the same as that used by the authors in a previous study [46]. Primary antibodies, used in this study are listed in Table 2. Antibodies were diluted in an antibody diluent (Dako, Glostrup, Denmark) and applied overnight at $4{ }^{\circ} \mathrm{C}$. The immunolabelling procedure included negative control sections incubated with PBS instead of the primary antibody. A mixture of biotinylated anti-mouse and anti-rabbit immunoglobulins (LSAB Kit; Dako), diluted in PBS, was used as secondary antibody, and applied for $30 \mathrm{~min}$. After washing in PBS, the sections were incubated in streptavidin conjugated to horseradish peroxidase in Tris- $\mathrm{HCl}$ buffer containing sodium azide (LSAB Kit; Dako) $0.015 \%$, for 30 min. To reveal immunolabelling, diaminobenzidine tetrahydrochloride was used as a chromogen, and haematoxylin was used as counterstain.

\section{Scoring of immunoreactivity}

The intensity of immunolabelling in each specimen, for each antibody, was scored by two independent observers (PM, MM) under blinded conditions, as performed in a previous study [47]. For each tumor 20 fields were examined at 200X magnification (20X objective 10X ocular), and immunosignal was scored from absent to strong, as follows: n.a., not assessed; - negative staining; +/- weak immunolabelling; + moderate immunolabelling; ++ extensive and strong immunolabelling.

\section{Protein extraction and SDS PAGE/Western blotting}

Six sarcoids (T1, T2, T3, T4, T5, T6), two samples of normal skin (N1, N2) and Hela cells line (positive control) were available for molecular analysis. Tissues were snap frozen in liquid nitrogen and homogenized in icecold lysis buffer (50 mM tris PH 7.5, $150 \mathrm{mM} \mathrm{NaCl}, 1 \%$ Triton, 0.25 \% Deoxicolic acid, $1 \mathrm{mM}$ EDTA) added with protease inhibitor cocktail (Sigma, Milan, Italy). HeLa cell lines were grown for 2 days in 60-mm dishes, washed with ice-cold phosphate saline buffer two times and lysed for $20 \mathrm{~min}$ in ice-cold lysis buffer. Tissue homogenates and cell lysates were clarified by centrifugation and protein concentration was determined by Bradford protein assay performed according to manufacturer protocol (Bio-Rad Laboratories, Milan, Italy). $50 \mu \mathrm{g}$ of total protein were boiled at $100^{\circ}$ for $5^{\prime}$ in Laemmli sample buffer (Bio-Rad Laboratories, Hercules, CA) and analyzed by SDS polyacrylamide gel electrophoresis (PAGE). The proteins were blotted from the gel onto nitrocellulose membranes. The membranes were blocked for $1 \mathrm{~h}$ with $5 \%$ bovine serum albumin (BSA) at room temperature and incubated with anti-MMP2 (1:500), anti-MMP9 (1:1000), anti-MMP14 (1:500), anti-TIMP-2 (1.200), anti-collagen I (1:5000), and anti-collagen III (1:5000) antibodies (Table 2). After appropriate washing steps, peroxidase-conjugated anti-rabbit or anti-mouse IgG (1:2000, Santa Cruz Biotechnology), were applied for $1 \mathrm{~h}$ at room temperature. After washing, bound

Table 2 List of primary antibodies used for immunohistochemistry and western blotting analysis

\begin{tabular}{|c|c|c|c|c|c|c|c|}
\hline Antibody & Manufacturer & Clone & Specificity & Host species & Antigen retrieval & IHC Dilution & WB Dilution \\
\hline VIMENTIN & Dako cytomation & V-9 & $\begin{array}{l}\text { man, cow, dog, hamster, } \\
\text { horse, rabbit, rat }\end{array}$ & mouse & No antigen retrieval & $1: 50$ & - \\
\hline SMOOTH MUSCLE ACTIN & Dako cytomation & $1-a-4$ & chicken, cow, rat & mouse & $\begin{array}{l}\text { Citrate, ph } 6.0,30 \mathrm{~min}, \\
\text { steamer }\end{array}$ & $1: 50$ & $1: 5000$ \\
\hline TYPE I COLLAGEN & Ab-cam & Col-1 & $\begin{array}{l}\text { rat, rabbit, cow, human, } \\
\text { pig, deer }\end{array}$ & mouse & $\begin{array}{l}\text { Citrate, ph } 6.0,30 \mathrm{~min} \text {, } \\
\text { steamer }\end{array}$ & $1: 100$ & $1: 5000$ \\
\hline TYPE III COLLAGEN & Millipore & le7-d7 & rabbit, human & mouse & $\begin{array}{l}\text { Citrate, ph } 6.0,30 \mathrm{~min} \text {, } \\
\text { steamer }\end{array}$ & $1: 100$ & $1: 5000$ \\
\hline MMP-2 & Thermo-scientific & $A b-7$ & human, mouse, rat, cow & rabbit & $\begin{array}{l}\text { Citrate, ph } 6.0,30 \mathrm{~min}, \\
\text { steamer }\end{array}$ & $1: 200$ & $1: 500$ \\
\hline MMP-9 & Millipore & $56-2 a 4$ & $\begin{array}{l}\text { human, rat, rabbit, } \\
\text { guinea pig }\end{array}$ & mouse & $\begin{array}{l}\text { Citrate, ph } 6.0,30 \mathrm{~min} \text {, } \\
\text { steamer }\end{array}$ & $1: 200$ & 1:1000 \\
\hline MT1-MMP (MMP-14) & Millipore & $113-5 b 7$ & human & mouse & $\begin{array}{l}\text { Citrate, ph 6.0, } 30 \mathrm{~min}, \\
\text { steamer }\end{array}$ & $1: 200$ & $1: 500$ \\
\hline TIMP-2 & Millipore & 2TMP05 & $\begin{array}{l}\text { bovine, guinea pig, human, } \\
\text { mouse, rat, rabbit }\end{array}$ & mouse & $\begin{array}{l}\text { Citrate, ph 6.0, } 30 \text { min, } \\
\text { steamer }\end{array}$ & $1: 200$ & $1: 200$ \\
\hline
\end{tabular}


antibody was visualized on enhanced chemiluminescence (ECL) film (Amersham Pharmacia Biotech). The blots were stripped and reprobed against mouse antiactin antibody (Santa Cruz Biotechnology) at 1:5000 to confirm equal loading of proteins in each lane. For collagen I and III protein detection the SDS-PAGE procedure was performed in non-denaturing conditions.

\section{Gelatinase zymography}

Substrate-specific zymography for determination of gelatinolytic activity of MMP-2 and MMP-9 was performed as previously described [48]. Briefly, $20 \mu \mathrm{g}$ of each protein extract were subjected to gel electrophoresis using $10 \%$ Zymogram (Gelatin) pre cast Gel and zymogram gel was developed according to manufacturer protocol (Bio-Rad Laboratories, Milan, Italy). After electrophoresis, the gel was washed twice $2.3 \%$ triton X-100 for $30 \mathrm{~min}$ and incubated in development buffer $(50 \mathrm{mM}$ tris, $200 \mathrm{mM} \mathrm{NaCl}, 5 \mathrm{mM} \mathrm{CaCl} 2,0.02 \%$ Brij- 35 at $37{ }^{\circ} \mathrm{C}$ for $20 \mathrm{~h}$. Gel was then stained with $0.5 \%$ coomassie blue R-250 in staining solution (40\% methanol, $10 \%$ acetic acid, $0.5 \%$ coomassie blue, $100 \mathrm{ml}$ deionized water) at room temperature (RT) for $1 \mathrm{~h}$ and was de-stained in destaining solution (40\% methanol, $10 \%$ acetic acid, $250 \mathrm{ml}$ deionized water), until clear lysis bands appeared. To quantify the intensities of the degradated bands, zymogram gel was scanned using ChemiDoc gel scanner (Bio-Rad Laboratories).

\section{Availability of supporting data}

The data sets supporting the results of this study are included in the article and its additional files.

\section{Abbreviations \\ ECM: extracellular matrix; MMP: matrix metalloproteinase; TIMP: tissue inhibitor of metalloproteinase; SFs: sarcoid fibroblasts; BPV-1: bovine papillomavirus type-1; BPV-2: bovine papillomavirus type-2; VIM: vimentin; a-SMA: alpha-smooth muscle actin; T: sarcoid samples; N: normal skin.}

\section{Competing interests}

The authors declare that they have no competing interests.

\section{Authors' contributions}

PM has conceived the study, coordinated the group and drafted the manuscript; MM together with PM, has participated in the conception and design of the study, in analysis and interpretation of histological and immunohistochemical data and drafted the manuscript; GB and BR have been involved in revising the manuscript for important intellectual content; $A C$ and MEDB carried out the molecular genetic studies, taking part in western blotting analysis, and zymography; AC helped with interpretation of molecular data and drafted the manuscript. All authors read and approved the final manuscript.

\section{Authors' information}

MM is employed as a researcher at the Department of Veterinary Medicine and Animal Production; BR and GB are employed as associate professors at the Department of Veterinary medicine and animal production; PM is employed as a full professor at the Department of Veterinary medicine and animal production; MEDB is a student trainee for the degree thesis; $A C$ is a post-doc research fellowship at Institute of Protein Biochemistry (IBP)National Research Council (CNR), Via Pietro Castellino 111, 80131 Naples, Italy.

\section{Acknowledgment}

The authors acknowledge Mr. Raffaele Ilsami for its technical support during microscopy analysis. This research was financially supported by Department of Veterinary Medicine and Animal Production, University of Naples, Italy.

\section{Author details}

'Department of Veterinary Medicine and Animal Productions, Naples University "Federico II", Via F. Delpino 1, 80137 Naples, Italy. 2Present Address: Institute of Protein Biochemistry (IBP) National Research Council (CNR), Via Pietro Castellino 111, 80131 Naples, Italy.

Received: 24 July 2015 Accepted: 28 January 2016

Published online: 02 February 2016

\section{References}

1. Borzacchiello G, Corteggio A. Equine sarcoid: state of the art. Ippologia. 2009;20:7-14.

2. Nasir L, Brandt S. Papillomavirus associated diseases of the horse. Vet Microbiol. 2013;167(Suppl 1-2):159-67.

3. Torrontegui BO, Reid SJ. Clinical and pathological epidemiology of the equine sarcoid in a referral population. Eq Vet Educ. 1994;6:85-8.

4. Nasir L, Campo MS. Bovine papillomaviruses: their role in the aetiology of cutaneous tumours of bovids and equids. Vet Dermatol. 2008;19:243-54.

5. Knottenbelt DC. A suggested clinical classification of the equine sarcoid. Clin Tech in Eq Pract. 2005;4:278-95.

6. Tarwid JN, Fretz PB, Clark EG. Equine sarcoids: A study with emphasis on pathological diagnosis. Compend Contin Educ. 1985;7:293-300.

7. Martens A, De Moor A, Demeulemeester J, Peelman L. Polymerase chain reaction analysis of the surgical margins of equine sarcoids for bovine papilloma virus DNA. Vet Surg. 2001;30:460-7.

8. Yuan Z, Gobeil PA, Campo MS, Nasir L. Equine sarcoid fibroblasts over-express matrix metalloproteinases and are invasive. Virol. 2010;396:143-51.

9. Mosseri S, Hetzel U, Hahn S, Michaloupoulou E, Sallabank HC, Knottenbelt DC, et al. Equine sarcoid: In situ demonstration of matrix metalloproteinase expression. Vet J. 2014;202(Suppl2):279-85.

10. Chambers G, Ellsmore VA, O'Brien PM, Reid SWJ, Love S, Campo MS, et al. Sequence variants of bovine papillomavirus E5 detected in equine sarcoids. Virus Res. 2003;96(Suppl 1-2):141-5.

11. Martens A, Moor ADE, Demeulemeester J, Ducatelle R. Histopathological characteristics of five clinical types of equine sarcoid. Res Vet Sci. 2000;69(Suppl3):295-300.

12. Cochrane AC. Models in vivo of wound healing in the horse and the role of growth factors. Vet Dermatol. 1997;8:259-72.

13. Hansen RR. Complications of EquineWound Management and Dermatologic Surgery. Vet Clin Eq. 2009;24:663-96.

14. Haddow A. Molecular repair, wound healing, and carcinogenesis: tumor production a possible overhealing? Adv Cancer Res. 1972;16:181-234.

15. Dvorak HF. Tumors: wounds that do not heal. Similarities between tumor stroma generation and wound healing. N Engl J Med. 1986;315:1650-9.

16. Schäfer M, Werner S. Cancer as an overhealing wound: an old hypothesis revisited. Nat Rev Mol Cell Biol. 2008;9 Suppl 8:628-38.

17. Imaizumi R, Akasaka Y, Inomata N, Okada E, Ito K, Ishikawa Y, et al. Promoted activation of matrix metalloproteinase (MMP)-2 in keloid fibroblasts and increased expression of MMP-2 in collagen bundle regions: implications for mechanisms of keloid progression. Histopathol. 2009;54(Suppl6):722-30.

18. Pilcher BK, Dumin JA, Sudbeck BD, Krane SM, Welgus HG, Parks WC. The activity of collagenase-1 is required for keratinocyte migration on a type I collagen matrix. J Cell Biol. 1997;137:1445-57.

19. Allen $\mathrm{DL}$, Teitelbaum $\mathrm{DH}$, Kurachi K. Growth factor stimulation of matrix metalloproteinase expression and myoblast migration and invasion in vitro. Am J Physiol Cell Physiol. 2003;284 Suppl 4:C805-15.

20. Giantin M, Aresu L, Benali S, Aricò A, Morello EM, Martano M, et al. Expression of matrix metalloproteinases, tissue inhibitors of metalloproteinases and vascular endothelial growth factor in canine mast cell tumours. I Comp Pathol. 2012:147 Suppl 4:419-29.

21. Osenkowski P, Toth M, Fridman R. Processing, shedding, and endocytosis of membrane type 1-matrix metalloproteinase (MT1-MMP). J Cell Physiol. 2004;200 Suppl 1:2-10.

22. Borzacchiello G, Mogavero S, De Vita G, Roperto S, Della Salda L, Roperto F. Activated platelet-derived growth factor beta receptor expression, PI3K-AKT 
pathway molecular analysis, and transforming signals in equine sarcoids. Vet Pathol. 2009;46 Suppl 4:589-97.

23. Brandt S, Tober R, Corteggio A, Burger S, Sabitzer S, Walter I, et al. BPV-1 infection is not confined to the dermis but also involves the epidermis of equine sarcoids. Vet Microbiol. 2011;150(Suppl 1-2):35-40.

24. Chambers G, Ellsmore VA, O'Brien PM, Reid SW, Love S, Campo MS, et al. Association of bovine papillomavirus with the equine sarcoid. J Gen Virol. 2003;84:1055-62.

25. Martens A, De Moor A, Ducatelle R. PCR detection of bovine papilloma virus DNA in superficial swabs and scrapings from equine sarcoids. Vet J. 2001:161:280-6

26. Otten N, von Tscharner C, Lazary S, Antczak DF, Gerber H. DNA of bovine papillomavirus type 1 and 2 in equine sarcoids: PCR detection and direct sequencing. Arch Virol. 1993;132:121-31.

27. Reid SW, Smith KT, Jarrett WF. Detection, cloning and characterisation of papillomaviral DNA present in sarcoid tumours of Equus asinus. Vet Rec. 1994;135:430-2.

28. Borzacchiello G, Russo V, Della Salda L, Roperto S, Roperto F. Expression of platelet-derived growth factor-b receptor and bovine papillomavirus E5 and E7 oncoproteins in equine sarcoid. J Comp Pathol. 2008;139:231-7.

29. Carr EA, Théon AP, Madewell BR, Griffey SM, Hitchcock ME. Bovine papillomavirus DNA in neoplastic and non-neoplastic tissues obtained from horses with and without sarcoids in the western United States. Am J Vet Res. 2001;62:741-4.

30. Nasir L, Reid SW. Bovine papillomaviral gene expression in equine sarcoid tumours. Virus Res. 1999:61:171-5.

31. Ragland WL, Spencer GR. Attempts to relate bovine papillomavirus to the cause of equine sarcoid: Equidae inoculated intradermally with bovine papillomavirus. Am J Vet Res. 1969;30:743-52.

32. Yuan ZQ, Gault EA, Gobeil P, Nixon C, Campo MS, Nasir L. Establishment and characterization of equine fibroblast cell lines transformed in vivo and in vitro by BPV-1: model systems for equine sarcoids. Virol. 2008;373:352-61.

33. Bogaert L, Martens A, De Baere C, Gasthuys F. Detection of bovine papillomavirus DNA on the normal skin and in the habitual surroundings of horses with and without equine sarcoids. Res Vet Sci. 2005;79 Suppl 3:253-8.

34. Yuan ZQ, Philbey AW, Gault EA, Campo MS, Nasir L. Detection of bovine papillomavirus type 1 genomes and viral gene expression in equine inflammatory skin conditions. Virus Res. 2007;124(Suppl1-2):245-9.

35. Wobeser BK, Hill JE, Jackson ML, Kidney BA, Mayer MN, Townsend HG, et al. Localization of Bovine papillomavirus in equine sarcoids and inflammatory skin conditions of horses using laser microdissection and two forms of DNA amplification. J Vet Diagn Invest. 2012;24 Suppl 1:32-41.

36. Williams IF, Heaton A, McCullagh KG. Connective tissue composition of the equine sarcoid. Eq Vet J. 1982;14 Suppl 4:305-10.

37. Bran GM, Goessler UR, Hormann K, Riedel F, Sadick H. Keloids: Current concepts of pathogenesis (Review). Int J Mol Med. 2009;24:283-93.

38. Theoret $\mathrm{CL}$, Olutoye $\mathrm{OO}$, Parnell LK, Hicks J. Equine exuberant granulation tissue and human keloids: a comparative histopathologic study. Vet Surg. 2013;42 Suppl 7:783-9.

39. Ries C, Pitsch T, Mentele R, Zahler S, Egea V, Nagase H, et al. Identification of a novel $82 \mathrm{kDa}$ proMMP-9 species associated with the surface of leukemic cells: (auto-) catalytic activation and resistance to inhibition by TIMP-1. Biochem J. 2007:405(Suppl3):547-58.

40. Mohan R, Chintala SK, Jung JC, Villar W, McCabe F, Russo L, et al. Matrix metalloproteinase gelatinase B (MMP-9) regulates and effects epithelial regeneration. J Biol Chem. 2002;277:2065-72.

41. OToole EA, van Koningsveld R, Chen M, Woodley DT. Hypoxia induces epidermal keratinocyte matrix metalloproteinase-9 secretion via the protein kinase C pathway. J Cell Physiol. 2008;214 Suppl 1:47-55.

42. Klein T, Bischoff R. Physiology and pathophysiology of matrix metalloproteases. Amino Acids. 2011;41(Suppl2):271-90.

43. Butler PD, Longaker MT, Yang GP. Current progress in keloid research and treatment. J Am Coll Surg. 2008;206 Suppl 4:731-41.

44. Bergvall KE. Sarcoids. Vet Clin North Am Eq Pract. 2013;29(Suppl3):657-71.

45. Pascoe RR, Knottenbelt DC. Manual of Eq Dermatol pub. London: WB Saunders; 1999. p. 244-50.
46. Restucci B, Martano M, Maiolino P. Expression of endothelin-1 and endothelin-1 receptor A in canine mammary tumours. Res Vet Sci. 2015;100:182-8.

47. Martano M, Carella F, Squillacioti C, Restucci B, Mazzotta M, Lo Muzio L, et al. Metallothionein expression in canine cutaneous apocrine gland tumors. Anticancer Res. 2012;32 Suppl 3:747-52.

48. Kleiner DE, Stetler-Stevenson WG. Quantitative zymography: detection of picogram quantities of gelatinases. Anal Biochem. 1994;218(2):325-9.

\section{Submit your next manuscript to BioMed Central and we will help you at every step:}

- We accept pre-submission inquiries

- Our selector tool helps you to find the most relevant journal

- We provide round the clock customer support

- Convenient online submission

- Thorough peer review

- Inclusion in PubMed and all major indexing services

- Maximum visibility for your research

Submit your manuscript at www.biomedcentral.com/submit
C Biomed Central 